Baoe Wang, Rihong Zhang*, Yiyong Li and Xiaoming Lian

\title{
CFD simulation of a swirling vortex cavitator and its degradation performance and pathway of tetracycline in aqueous solution
}

https://doi.org/10.1515/ijcre-2021-0243

Received September 20, 2021; accepted January 27, 2022; published online February 11, 2022

\begin{abstract}
The vortex cavitation technology shows a great development potential for antibiotic wastewater treatment. We have designed a novel swirling vortex cavitator for tetracycline degradation in aqueous solution. By Computational Fluid Dynamics (CFD) simulation using ANSYS CFX flow field calculation software, it was found that the vortex cavitator could form a low-pressure area far lower than the saturated vapor pressure at experimental temperature, resulting in generation of obvious vortex cavitation effect, which also proved by the concentration of hydroxyl radical of $4.58 \mu \mathrm{mol} / \mathrm{L}$ measured by methylene blue method. Moreover, the tetracycline degradation process may be mainly caused by the oxidation of hydroxyl radicals. The batch experiments results showed when the degradation time was $30 \mathrm{~min}$, the vortex cavitator had a good degradation ability of tetracycline in $\mathrm{pH}$ range of 5.0-9.0. The degradation efficiency of $2.0 \mathrm{mg} / \mathrm{L}$ tetracycline solution was $76.45 \%$ within $50 \mathrm{~min}$ at $\mathrm{pH}$ 7.0. The possible intermediate products of tetracycline were determined by HPLC-MS Spectrometry. A series of reactions including hydroxylation, decarbonylation and $\mathrm{C}-\mathrm{N}$ bond cleavage, ring-opening, and removing two methyl groups in the carbon atom ring, occur during the degradation.
\end{abstract}

Keywords: computational fluid dynamics; degradation; Swirling vortex cavitator; tetracycline.

\section{Introduction}

Antibiotics are commonly utilized for treatment and prevention of deadly infections in humans and animals. In the

\footnotetext{
*Corresponding author: Rihong Zhang, College of Mechanical and Electrical Engineering, Zhongkai University of Agriculture and Engineering, Guangzhou 510225, China, E-mail: zrh-neu@163.com Baoe Wang, Yiyong Li and Xiaoming Lian, College of Resource and Environment, Zhongkai University of Agriculture and Engineering, Guangzhou 510225, China
}

mid-1990s, researchers have detected human or veterinary antibiotic residues in a variety of substrates on the earth. These residual antibiotics will threaten ecology and human health due to high eco-toxicity. It is of great significance to study the treatment technology of antibiotics for controlling the source of antibiotics in the environment (Brausch et al. 2012). Various antibiotic wastewater treatment technologies have been reported, such as photocatalysis technology (Li et al. 2020; Wei, Liu, and Shangguan 2020), adsorption (Manuel et al. 2020; Wang et al. 2020), microalgae biological treatment technology (Leng et al. 2020) and other advanced oxidation technologies like Fenton and Fenton-like process, ozonation or catalytic ozonation, and electrochemical oxidation (Wang and Zhuan 2020). Among these, advanced oxidation technology shows great development potential for antibiotic wastewater treatment (Anjali and Shanthakumar 2019). However, due to the high cost of advanced oxidation technology for the treatment of antibiotic wastewater, it is not applied to wastewater treatment engineering at present. Thus, it is urgent to develop new treatment technology for antibiotic wastewater treatment.

Hydrodynamic cavitation technology has been emerged to deal with wastewater coming from various industries (Simpson and Ranade 2018). Cavitation effect is a rapid phase-change phenomenon in liquid, which is the process of bubble formation, development and collapse when the pressure of liquid falls below the vapor pressure of that liquid. This collapsing act generates transient temperature in the range of 8000-10,000 K and pressures of 800-1000 atm. Such extreme conditions can generate enough energy to interrupt the molecular chain of organic matter locally, or form $\cdot \mathrm{OH}$ and $\cdot \mathrm{H}$ radicals, which act as an oxidant for the degradation of pollutant present in the wastewater (Wang et al. 2011).

Many kinds of organic compounds in water, including benzene (Thanekar et al. 2021), dichlorvos (Joshi and Gogate 2012), reactive orange 4 (Gore et al. 2014), chloroform (Franke et al. 2011) and sulfadiazine (Kuldeep and Moholkar 2020) can be degraded efficiently by hydrodynamic cavitation. The hydrodynamic cavitation technology includes vortex cavitation technology and jet cavitation technology. Compared with vortex cavitation technology, 
jet cavitation requires more complex equipment and more energy consumption because the fluid must obtain a very high initial velocity before entering the jet device. Vortex can form cavitation under the condition of low inlet pressure. Moreover, Vortex devices offer various advantages like early inception, high cavitation yield and significantly lower propensity to clogging and erosion (Vivek et al. 2021). Therefore, the vortex cavitation technology shows great potential and application prospect in the treatment of toxic substance and refractory organics.

Different types of vortex cavitation devices have been designed (Chahine and Kalumuck 2001; Curt and Morten 2012), but the degradation efficiency of organic compounds by certain vortex cavitation devices is not ideal (Wang et al. $2008,2020)$. This technology has not yet been fully realized in practice. Some scholars think that there are mainly two reasons: one is designs of cavitation reactor, and the other is lack of systematic design, optimization and scale-up methodologies (Sarvothaman, Simpson, and Ranade 2019). Therefore, we designed a novel type of swirling vortex cavitator. Its combination structure of vortex cavity and spiral flow passage distributed small holes is novel and the device structure has been obtained patent license of China (Wang, Zhang, and Lian 2019). In this study, the Computational Fluid Dynamics (CFD) simulation analysis was carried out to demonstrate the generation of vortex cavitation effect. The degradation performance of antibiotics in aqueous solution under different conditions was discussed. Furthermore, the possible degradation pathway of tetracycline was proposed.

\section{Materials and methods}

\subsection{Tetracycline}

Tetracycline (TC), $\mathrm{C}_{22} \mathrm{H}_{24} \mathrm{~N}_{2} \mathrm{O}_{8}$ (Shanghai Yuanye Biotechnology Co., Ltd.), is analytical pure $98 \%$.

\subsection{Flow field simulation software}

ANSYS CFX simulation software is utilized to simulate and analyze the vortex cavitator, and Creo 3D design software is used to create the model of key vortex cavitator position.

\subsection{CFD simulation methods}

2.3.1 CFD simulation models: CFD simulation applies Mixture model of multiphase flow model, basic fluid model and Realizeble $k-\varepsilon$ doubleequation turbulence model. Mass transfer in Mixture model considers cavitation effect. The model assumes that the influence of temperature and the heat transfer process in the flow is ignored. Assumption of the initial fluid state is that the fluid medium is stationary.

2.3.2 Boundary conditions setting: Boundary conditions are as follows: The inlet boundary condition adopts pressure inlet boundary condition, and the total pressure of the inlet is set as $0.3 \mathrm{Mpa}$. The outlet boundary condition adopts pressure outlet boundary condition, and the back pressure of the outlet is set as $0 \mathrm{MPa}$. The operating pressure is set as one atmosphere (101,325 $\mathrm{Pa})$. The medium of the fluid is set as "water", and the boundary condition is set as "water liquid".

2.3.3 Meshing of vortex cavitation model: In view of the complex fluid field structure, many positions of corner, a large number of holes and small boundaries, the meshing of vortex cavitation model adopts unstructured grid technology of software Gambit. The wall part of cavitator arranges boundary layer network. The grid was dense in the region of severe regional changes in geometry and within the scope of general and cavitation processing. Large scale grid cell is used in the region of not severe changes of flow.

2.3.4 Sensitivity analysis and solution convergence: In CFD simulation, DELL-T7910 desktop workstation was used for calculation, equipped with E5-2600 V3 processor, memory capacity of $32 \mathrm{~GB}$ and video memory of $4 \mathrm{~GB}$. The average computing time is $51 \mathrm{~min}$. Convergence calculation results were obtained. The grid scale was directly given to 2,124,961 according to experience.

The convergence standard is the default $10 \mathrm{E}^{-3}$ convergence standard, and the calculation is converged.

\subsection{Experiments methods}

2.4.1 Cyclic degradation process by swirling vortex cavitator: The cyclic degradation process is shown in Figure 1. It is a closed loop

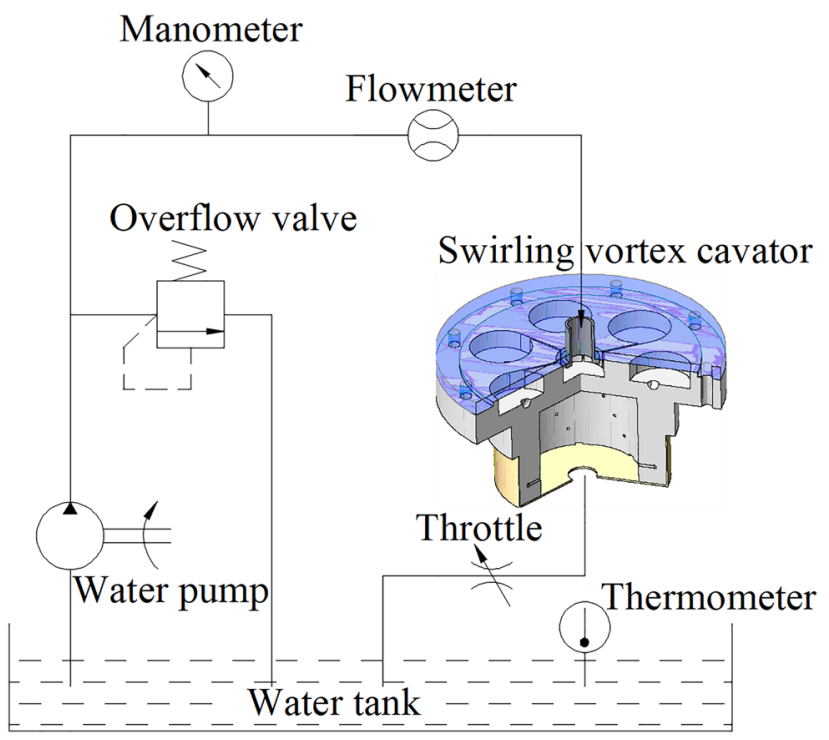

Figure 1: Cyclic degradation process by the swirling vortex cavitator. 
system designed to pump $10 \mathrm{~L}$ of solution from water tank, then taking it into the swirling vortex cavitator and discharging the treated solution back to the water tank. The overflow valve is used for adjusting the inlet liquid pressure of the swirling vortex cavitator.

2.4.2 Tetracycline degradation by swirling vortex cavitator: Tetracycline solution of $10 \mathrm{~L}$ with an initial concentration of $2.0 \mathrm{mg} / \mathrm{L}$ adjusted $\mathrm{pH}$ in advance was placed in the water storage tank. Eight ice bags were placed to prevent the solution temperature from being too high. Then the water pump was started (the inlet pressure of the pump was set to be $0.15 \mathrm{MPa}$ ) and the degradation began. The ice bags were replaced at degradation time $30 \mathrm{~min}$. The sample of $2.0 \mathrm{~mL}$ tetracycline solution was taken and filtered into the chromatographic bottle with $0.22 \mu \mathrm{M}$ filter membrane for determination the concentration of tetracycline every certain time. After the degradation, rinsed the water pump and water tank three times with deionized water.

The batch degradation experiments were conducted under the basic conditions, which are initial solution $\mathrm{pH}$ 7.0, initial tetracycline concentration $2.0 \mathrm{mg} / \mathrm{L}$, degradation time $30 \mathrm{~min}$, and inlet pressure of the pump $0.15 \mathrm{MPa}$, except for the change of the value of this parameter in an influence experiment.

All of the experiments were performed at least in triplicates.

2.4.3 Analytical methods: The concentrations of tetracycline were analyzed by HPLC. It was performed in a WATER 2695 HPLC instrument using a Extend-C18 column $(150 \mathrm{~mm} \times 4.6 \mathrm{~mm}, 3.5 \mu \mathrm{m}$ particle size). The intermediate products analysis of degradation was performed in an Agilent 1290/6470 HPLC-MS instrument using an Agilent column ( $50 \mathrm{~mm} \times 4.6 \mathrm{~mm}, 2.1 \mu \mathrm{m}$ particle size). The spectrometry analysis software was SmartDalton HR software (Guangzhou Molecular Information Company). NIST2020, Wiley11 and Elsevier databases were for spectral comparison.

2.4.4 Determination of hydroxyl radical concentration: Methylene blue method was used to determine the concentration of hydroxyl radical produced by the vortex cavitator (Zhang, Yang, and Li 2007), and the calculation formula is as follows:

$$
C_{\text {. } \mathrm{OH}}=\frac{\Delta A}{0.07196}
$$

where, $C_{\bullet \mathrm{OH}}$ is the molar concentration of hydroxyl radical, $\mu \mathrm{mol} / \mathrm{L}$. $\Delta A$ is absorbance difference of methylene blue in solution before and after degradation $\left(\lambda_{\max }=664 \mathrm{~nm}\right)$.

\subsection{Degradation efficiency}

The degradation efficiency of tetracycline is calculated by the equation as follows:

$$
X=\frac{C_{0}-C_{1}}{C_{0}} \times 100 \%
$$

where $C_{0}$ is the initial concentration of tetracycline, mg/L. $C_{1}$ is the concentration of tetracycline after degradation, $\mathrm{mg} / \mathrm{L}$. The concentration of tetracycline is determined by HPLC.

\section{Results and discussion}

\subsection{Vortex cavitation effect generation analysis and CFD simulation of swirling vortex cavitator}

\subsubsection{Vortex cavitation effect generation analysis of the swirling vortex cavitator}

The three-dimensional section view of the designed swirling vortex cavitator is shown in Figure 2. The swirling vortex cavitator is mainly composed of an inlet joint, an upper end cover, a vortex cavitation cavity, a jet cavity and a baffle below. The main vortex cavitation part includes six vortex cavities and six spiral flow passages distributed four small holes each flow passage. Six vortex cavities are designed in the vortex cavitator in order to make full use of the geometric structure, form more vortex cavitation effect and improve cavitation efficiency. After all, each vortex cavity can play the role of contributing vortex cavitation effect generation.

The structural dimensions of the swirling vortex cavitator are as follows: the diameter of the vortex cavity is $65 \mathrm{~mm}$. The width of the vortex cavity gap is $5 \mathrm{~mm}$. The diameter of the inlet of the flow passage is $9 \mathrm{~mm}$, and the diameter of the outlet of the flow passage is $3 \mathrm{~mm}$. The number of holes in each spiral flow passage is 4 and the diameter is $3 \mathrm{~mm}$.

As can be seen from the structure diagram of the swirl vortex cavitator (Figure 2), the fluid enters the center of the vortex cavitation component after passing through the

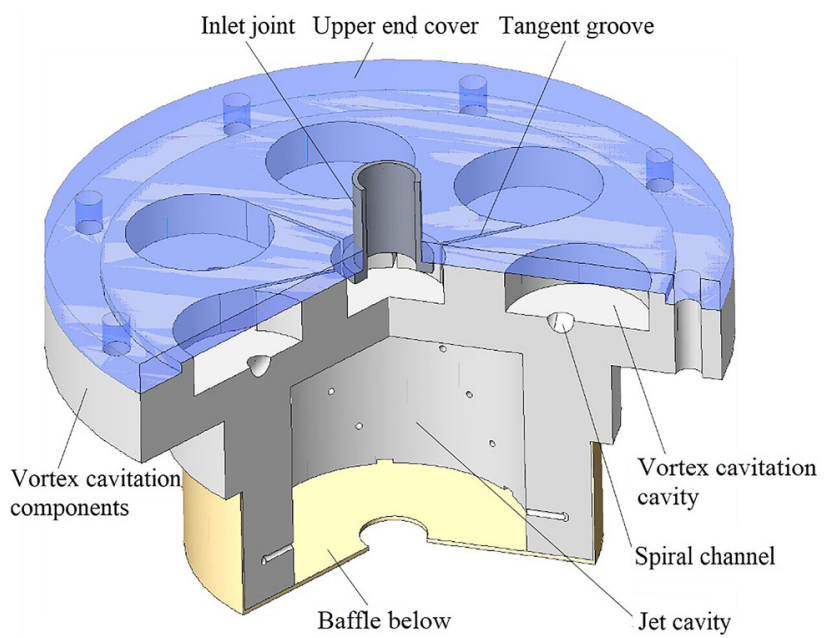

Figure 2: Three-dimensional sectional structure of the swirling vortex cavitatior core components. 
inlet above the upper end cover, and then diverges. Under the action of the slit in the center edge of the vortex cavitation unit, the fluid will generate a large inlet velocity and enter the respective vortex cavity. The fluid will swirl around the swirling cavity and enter the spiral flow passage. The cross section of the spiral flow passage is designed gradual decrease with the direction of the flow. According to the relationship between flow velocity and flux area, the fluid will produce a very high flow velocity at the outlet of the flow passage. Combining with Bernoulli equation, it can be concluded that local low pressure is formed here and cavitation bubbles are formed accordingly. After running out of the flow passage, the crosssectional area rises sharply and the pressure recovery. The internal and external pressure of the cavitation bubbles cannot maintain its shape, resulting in collapse of cavitation bubbles and cavitation effect generation. Each vortex cavity is designed with a spiral flow passage leading to the jet cavity, and the fluid in multiple flow passages is driven to collide violently with the baffle designed below the vortex components, so that the small bubbles collapse more thoroughly, thus improving the cavitation effect. In order to generate more times of cavitation, four holes leading to the jet cavity are distributed on each spiral flow passage to create more conditions for cavitation effect generation.

\subsubsection{CFD simulation of the swirling vortex cavitator}

The 3D model of swirling vortex cavitator was created by using Creo 3D design software (shown in Figure 3). Figure 4 displays the mesh of fluid region of vortex cavitation. Figure 5 shows the streamline distribution diagram of the fluid in the vortex cavitator. We can see the movement of the fluid in the device from the figure. The fluid enters the vortex cavity through the gap above the vortex cavitator, carries out rotating movement in the vortex cavity, and then enters the spiral flow passage.

Figure 6 shows the fluid pressure distribution diagram of the simulated vortex cavitator. The low-pressure area is mainly distributed in the jet cavity, where a certain number of cavitation bubbles will be generated. It can be seen from the pressure cloud map that the minimum pressure of the device fluid is about 5,150 $\mathrm{Pa}$. The test temperature is controlled below $55^{\circ} \mathrm{C}$, and the saturated vapor pressure of pure water at $55^{\circ} \mathrm{C}$ is $15,737 \mathrm{~Pa}$. Thus, the minimum pressure value generated by the vortex cavitation device is far lower than the saturated vapor pressure at this temperature. Therefore, obvious vortex cavitation effect can be produced under the test temperatures.

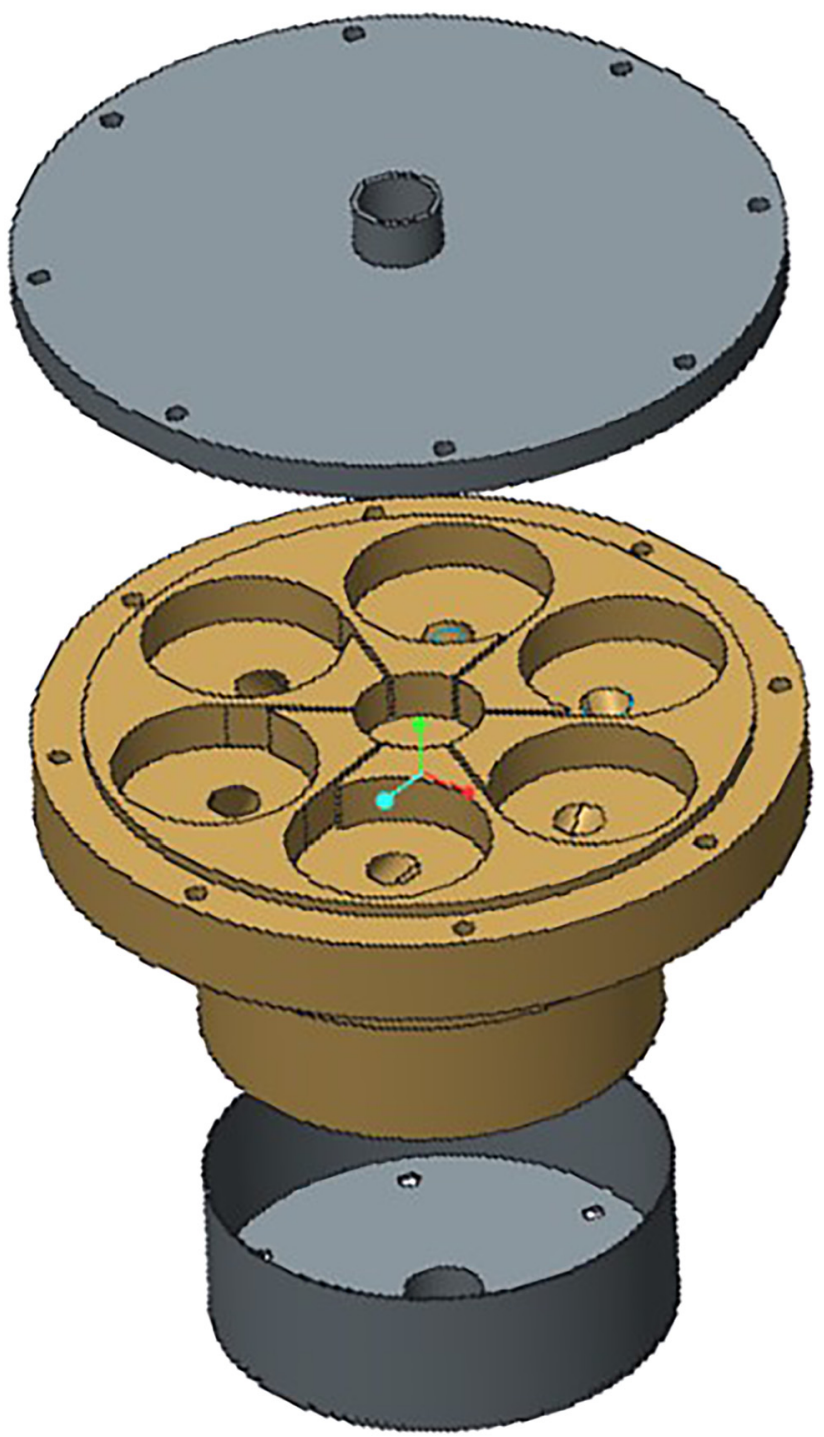

Figure 3: Three-dimensional structure of the swirling vortex cavitator.

By comparing the pressure cloud map in Figure 5 with the fluid flow diagram in Figure 6, it can be found that a local low-pressure area will be formed before the fluid is rushed out from the outlet of the flow passage. When the fluid flows out through the outlet, the cross-sectional area rises sharply. At this time, the internal and external pressure of the cavitation bubble cannot maintain its shape due to the pressure recovery, resulting in collapse, and then cavitation effect occurs.

\subsection{Detection of hydroxyl radical in vortex cavitator}

In general, the mechanism of vortex cavitation degradation of organic matter is due to the strong oxidation of hydroxyl 


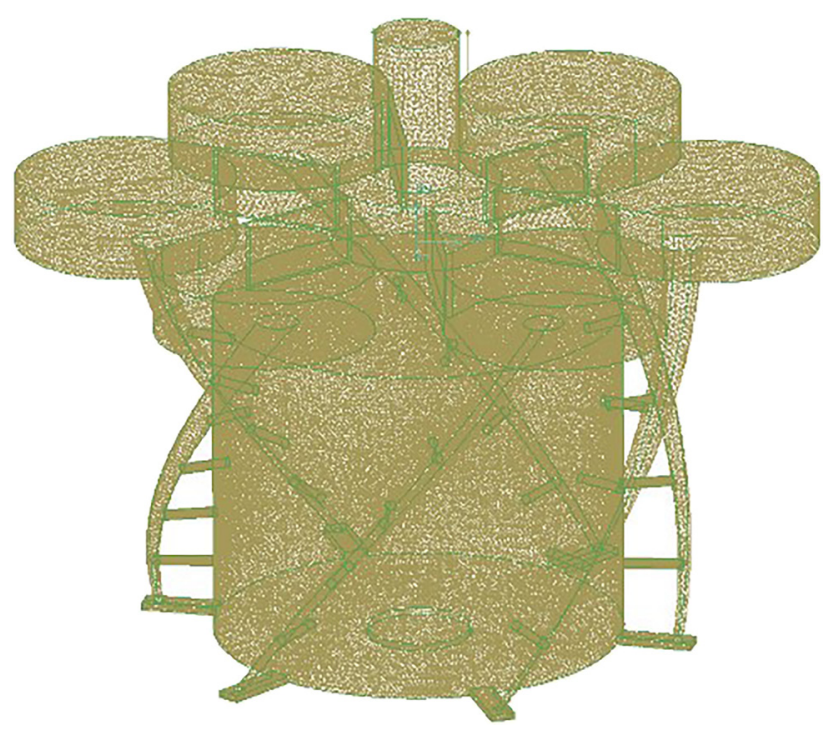

Figure 4: Mesh model of fluid region.

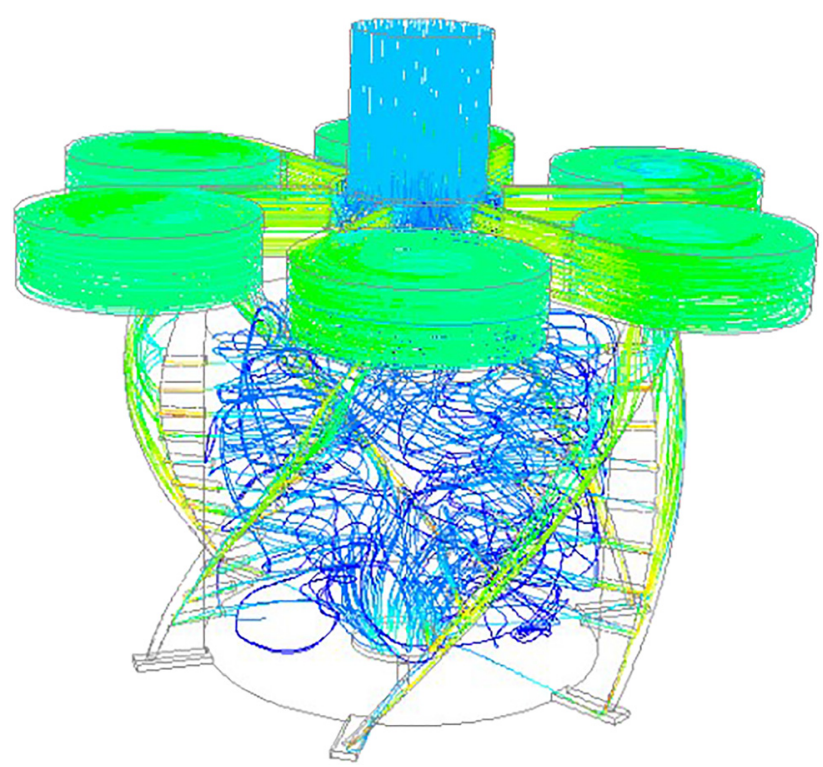

Figure 5: Flow line distribution of the swirling vortex cavitator.

radicals produced by vortex cavitation effect. Direct $\bullet \mathrm{OH}$ quantification is however unlikely due to their high reactivity and short life-time (circa $10^{-9} \mathrm{~s}$ ). Methylene blue method was used to measure indirectly the concentration of hydroxyl radical at different times, and the results are shown in Figure 7.

As can be seen from Figure 7, our designed swirling vortex can generate hydroxyl radicals. As the concentration of methylene blue solution increased, the capture rate of hydroxyl radicals increased. When the concentration of methylene blue solution reached $20 \mathrm{mg} / \mathrm{L}$, the capture rate of hydroxyl radical was unchanged, which indicated that the hydroxyl radical produced by vortex cavitation have been fully captured by methylene blue. The maximum concentration of hydroxyl radical was $4.58 \mu \mathrm{mol} / \mathrm{L}$, which was far more than that reported by other cavitation device (Zhang, Yang, and Li 2007). It can concluded that the swirling vortex cavitator can produce a high concentration of hydroxyl radicals, and the degradation of tetracycline is mainly completed under the oxidation of hydroxyl radicals.

\subsection{Degradation of tetracycline at different initial $\mathrm{pH}$ values}

The performance of tetracycline degradation by the vortex cavitator in the range of initial $\mathrm{pH}$ of tetracycline solution 5.0-9.0 and at degradation time $30 \mathrm{~min}$ is shown in Figure 8.

As can be seen from Figure 8, the degradation efficiency of tetracycline solution reached the highest of $60.93 \%$ at $\mathrm{pH}$ 7.0. It indicated that vortex cavitation effect was beneficial to the degradation of tetracycline solution under neutral conditions. Although the acid conditions are conducive to the generation of hydroxyl radicals, tetracycline will be converted into a difficulty degradation construction of heterotropic tetracycline, resulting in a decrease of the total degradation efficiency in acid conditions. Alkaline conditions inhibit the generation of hydroxyl radicals. However, the degradation efficiency of tetracycline in this device under initial pH 5.0 to 9.0 is more than $49 \%$. It indicated that the vortex cavitator has a good degradation ability on tetracycline in this $\mathrm{pH}$ range. Moreover, the degradation efficiency of tetracycline is higher than that by jet hydrodynamic cavitation reported by Wang, Jia, and Wang (2017).

\subsection{Degradation of tetracycline at different initial concentrations of tetracycline}

Figure 9 shows the degradation rate of tetracycline by the vortex cavitator in the range of initial concentration of tetracycline $2.0-10.0 \mathrm{mg} / \mathrm{L}$.

The degradation efficiency of tetracycline decreased gradually with the increase of the initial concentration of tetracycline. It gradually decreased from 60.93 to $34.92 \%$ with the concentration of tetracycline ranged from 2 to $10 \mathrm{mg} / \mathrm{L}$. It may due to the constant amount of hydroxyl radicals generated by vortex cavitation effect. When the initial concentration of tetracycline increases, the degradation efficiency of tetracycline decreases accordingly due 


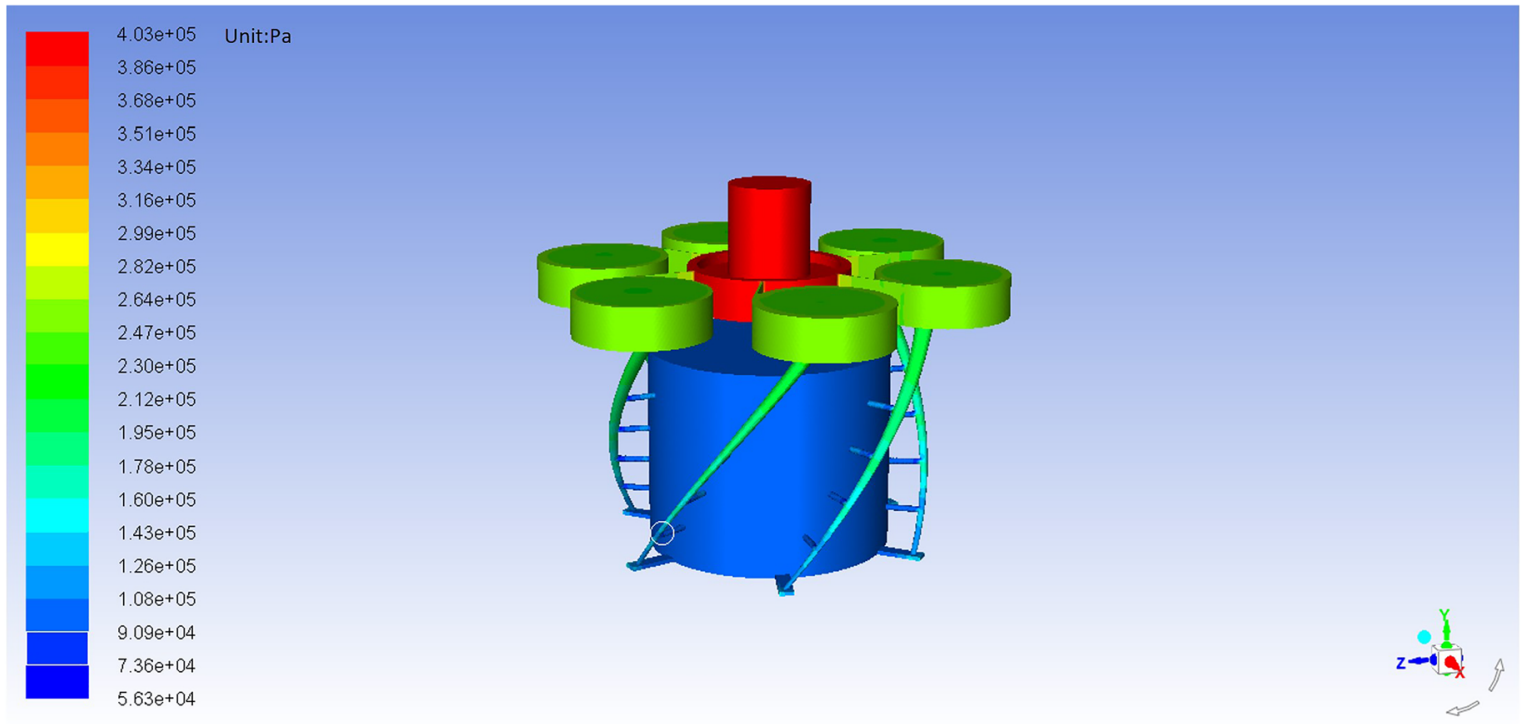

Figure 6: Fluid pressure cloud of the swirling vortex cavitator.

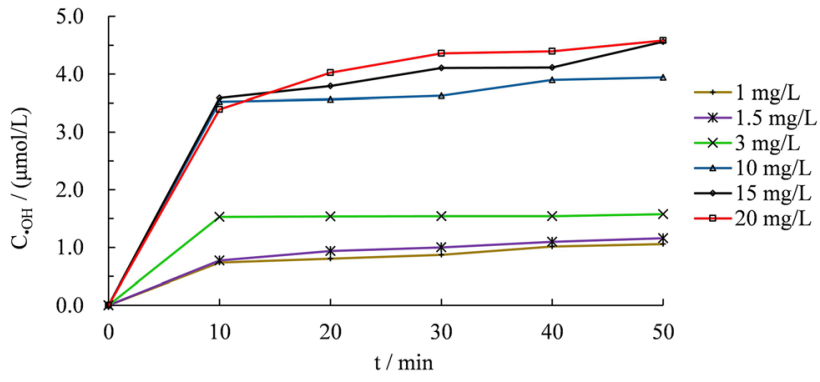

Figure 7: The concentration of hydroxyl radicals generated by the swirling vortex cavitator at different methylene blue concentrations.

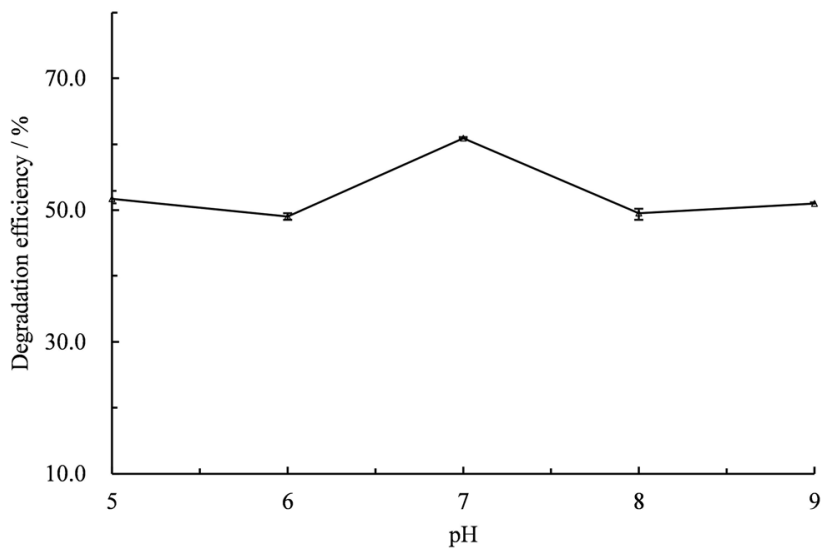

Figure 8: Degradation efficiency of tetracycline at different $\mathrm{pH}$.

to the limited amount of tetracycline degradation. Nevertheless, the absolute degradation amount of tetracycline at higher initial concentration of tetracycline was larger than

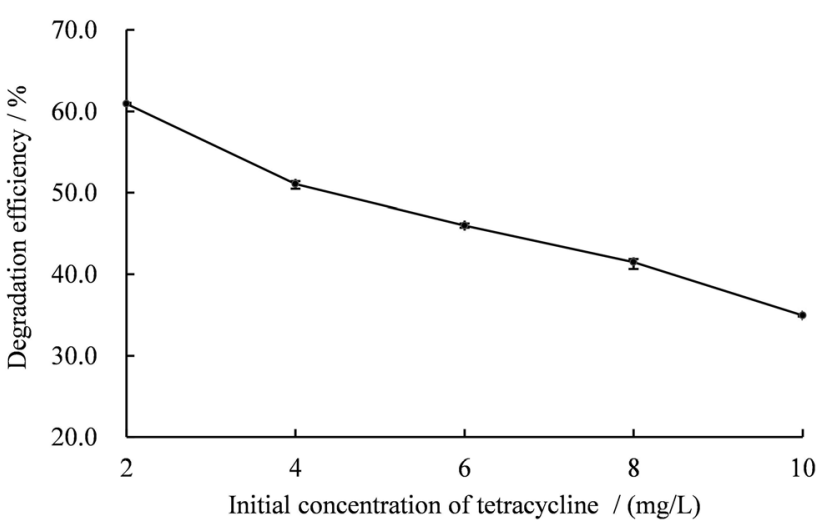

Figure 9: Degradation efficiency of tetracycline at different concentrations of tetracycline.

that of tetracycline at low concentration owing to the fuller utilization of hydroxyl radicals.

\subsection{Degradation of tetracycline at different reaction times}

Figure 10 shows the degradation efficiency of tetracycline by the vortex cavitator at different degradation times.

The degradation efficiency of tetracycline in solution increased with reaction time gradually. It reached 33.97\% within $10 \mathrm{~min}$, and the highest $74.27 \%$ after 50 min reaction. However, the degradation rate of tetracycline solution decreased gradually. The reason can be analyzed from system temperature and the intermediates of degradation. 


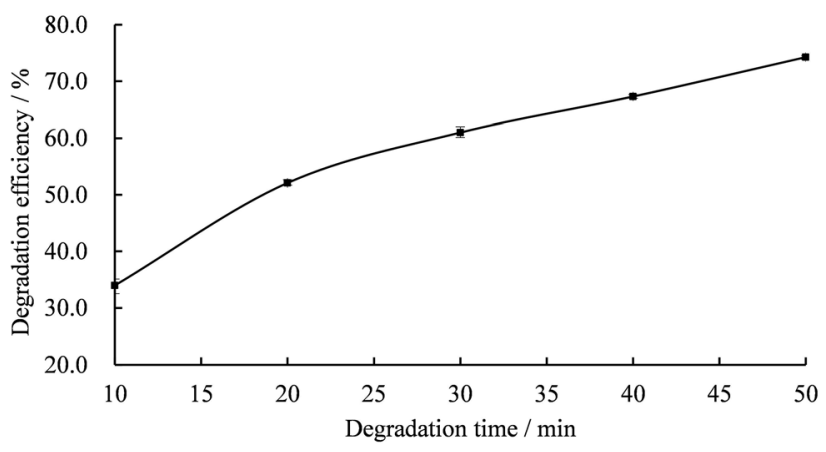

Figure 10: Degradation efficiency of tetracycline at different degradation times.

Firstly, at the beginning of the reaction, a large number of hydroxyl radicals produced by vortex cavitation effect reacted with tetracycline quickly. But as the reaction time went on, degradation process of tetracycline would gradually accumulate intermediates, which would compete with tetracycline combined with hydroxyl radicals. Secondly, the temperature of tetracycline solution would increase with the reaction time. The increase temperature has a double effect on degradation. On one hand, the saturated vapor pressure of the solution increases with the increase of temperature. Accordingly, the different pressure between the fluid pressure and the saturated vapor pressure becomes larger with the increase of temperature, which makes it easier to form the pressure difference required for the formation of vortex cavitation effect. At the same time, the increase of temperature is also conducive to promoting the hydrolysis of tetracycline. On the other hand, there are a certain number of gas cores dissolved in the solution in the natural state. The number of gas cores are proportional to the intensity of vortex cavitation effect. But the number of gas cores decreases with the increase of temperature. Therefore, there is an optimum temperature for the intensity of vortex cavitation effect. Thus, the degradation gradually slowed down over time combined with the above influence factors.

\subsection{Analysis of degradation pathway of tetracycline}

TC is consisting of connected rings with various ionizable functional groups as well as electron-rich moieties (dimethyl amino group, phenolic group, and conjugated double bond) (Yang et al. 2021). In the process of vortex cavitation, huge energy and large amount of $\cdot \mathrm{OH}$ is produced, which can react with tetracycline, such as cracking, oxidation reaction. The possible intermediate products of tetracycline (after 30 min degradation) were determined by HPLC-MS Spectrometry. Some new peaks appeared in the degradation intermediates, such as $m / z=462, \mathrm{~m} / z=401$, $m / z=376, m / z=306, m / z=278, m / z=149$. The possible degradation pathway of tetracycline was proposed in Figure 11. The product $\mathrm{TC}_{1}(m / z=462)$ was identified as the hydroxylation of TC (Niu et al. 2021). This is an oxidation

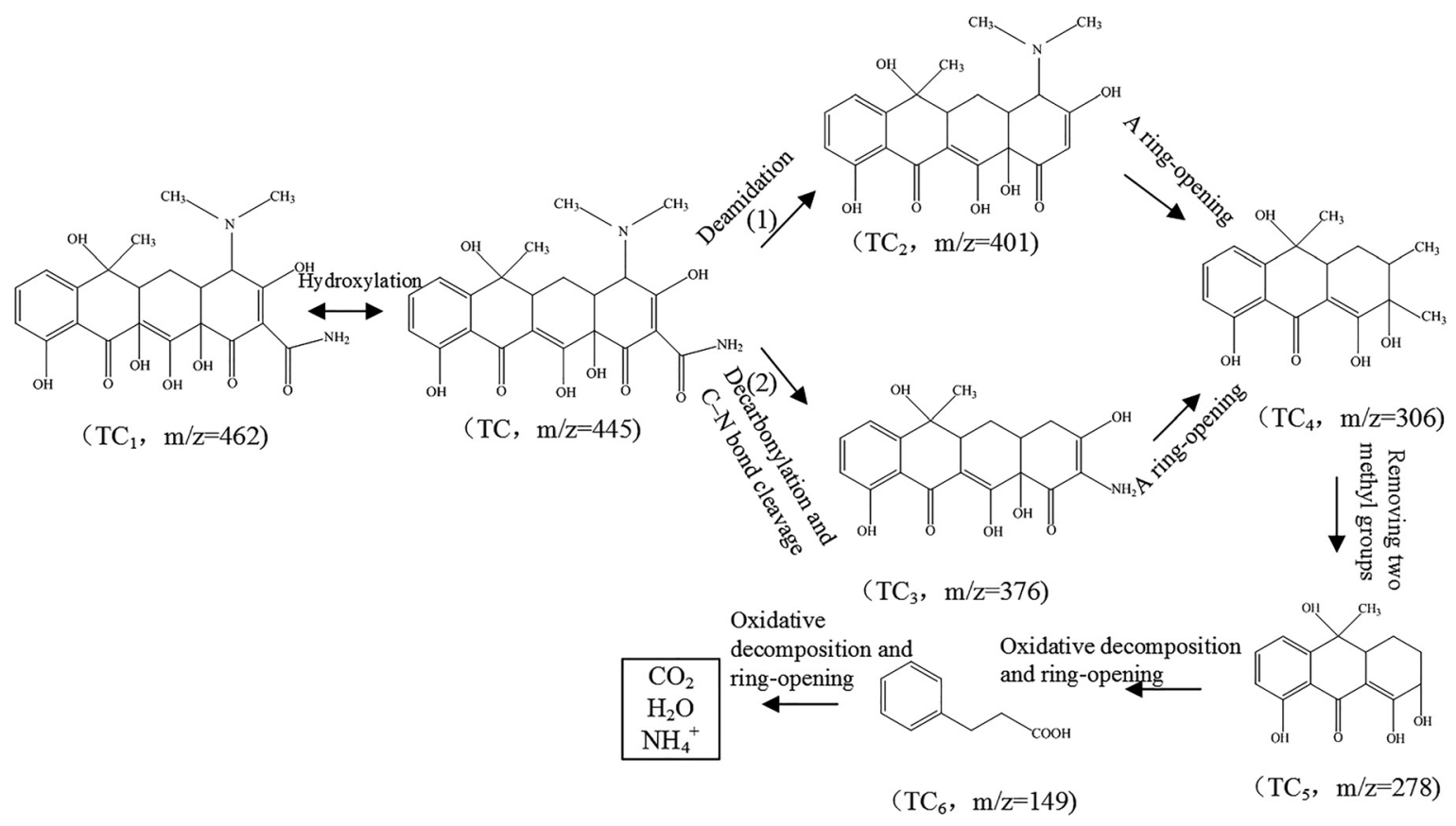

Figure 11: Possible degradation pathway of tetracycline. 
product (Chiarello et al. 2016). The reason is that $-\mathrm{C}=\mathrm{C}-$ conjugates with the oxygen on the adjacent $-\mathrm{OH}$. As an electron donor, $-\mathrm{OH}$ weakens the connection between $\mathrm{C}=\mathrm{C}-$, making it an easy site to be attacked by $-\mathrm{OH}$ (Khan, Bae, and Jung 2010). $\mathrm{TC}_{2}(m / z=401)$ is deamidation of tetracycline molecules ( $\mathrm{Zhu}$ et al. 2021). At the same time, the decarbonylation and $\mathrm{C}-\mathrm{N}$ bond cleavage of $\mathrm{TC}$ forms an intermediate with $\mathrm{m} / z 376\left(\mathrm{TC}_{3}\right)$. A ring-opening reaction occurred in the products $\mathrm{TC}_{2}$ and $\mathrm{TC}_{3}$ to obtain an intermediate of $\mathrm{TC}_{4}(m / z=306)$ (Guo et al. 2020). The product $\mathrm{TC}_{5}(m / z=278)$ was detected by removing two methyl groups in the carbon atom ring under large energy generated by vortex cavitation effect. $\mathrm{TC}_{5}$ continuously decomposed to aromatic compounds $\mathrm{TC}_{6}(m / z=149)$. With increasing reaction time, through oxidative decomposition and ring-opening reactions, the $\mathrm{TC}_{6}$ is finally decomposed into $\mathrm{H}_{2} \mathrm{O}, \mathrm{CO}_{2}$ and $\mathrm{NH}_{4}{ }^{+}$, etc.

\section{Conclusions}

The designed swirl vortex cavitator can form a low-pressure area far lower than the saturated vapor pressure. It can produce obvious vortex cavitation effect. When the degradation time was $30 \mathrm{~min}$, the vortex cavitator had a good degradation ability of tetracycline in $\mathrm{pH}$ range of 5.0-9.0. The degradation efficiency of $2.0 \mathrm{mg} / \mathrm{L}$ tetracycline was more than $49 \%$ at this $\mathrm{pH}$ range. The degradation rate decreased with the increase of tetracycline concentration, and the degradation rate was higher in $10 \mathrm{~min}$. The degradation efficiency of tetracycline solution was $74.27 \%$ in $50 \mathrm{~min}$.

The concentration of hydroxyl radical produced by swirl vortex cavitation was $4.58 \mu \mathrm{mol} / \mathrm{L}$, and the degradation of tetracycline may be mainly completed under the strong oxidation of hydroxyl radical. A series of reactions including hydroxylation, decarbonylation and $\mathrm{C}-\mathrm{N}$ bond cleavage, ring-opening, and removing two methyl groups in the carbon atom ring, occur during the degradation.

Author contributions: All the authors have accepted responsibility for the entire content of this submitted manuscript and approved submission.

Research funding: The authors are thankful to Agriculture and Rural Affairs Department of Guangdong Province for providing financial support (two million CNY) in the form of Project of Agricultural Scientific Research and Agricultural Technology Extension of Guangdong Province (2021KJ271), and to Science and Technology Planning Project of Guangdong Province (2016A020210126).
Conflict of interest statement: The authors declare no conflicts of interest regarding this article.

\section{References}

Anjali, R., and S. Shanthakumar. 2019. "Insights on the Current Status of Occurrence and Removal of Antibiotics in Wastewater by Advanced Oxidation Processes." Journal of Environmental Management 246: 51-62.

Brausch, J. M., K. Connors, B. W. Brooks, and G. M. Rand. 2012. "Human Pharmaceuticals in the Aquatic Environment: A Review of Recent Toxicological Studies and Considerations for Toxicity Testing." Reviews of Environmental Contamination \& Toxicology 218: 1-99.

Chahine, G. L., and K. M. Kalumuck. 2001. Swirling Fluid Jet Cavitation Method and System for Efficient Decontamination of Liquids. United States Patent. US6221260B1.

Chiarello, M., L. Minetto, S. V. Della Giustina, L. L. Beal, and S. Moura. 2016. "Popular Pharmaceutical Residues in Hospital Wastewater: Quantification and Qualification of Degradation Products by Mass Spectroscopy After Treatment with Membrane Bioreactor." Environmental Science \& Pollution Research 23: 16079-89.

Curt, H. T., and O. M. Morten. 2012. Vortex Generator with Vortex Chamber. United States Patent. US 20120097280A1.

Franke, M., P. Braeutigam, Z. L. Wu, Y. Ren, and B. Ondruschka 2011. "Enhancement of Chloroform Degradation by the Combination of Hydrodynamic and Acoustic Cavitation." Ultrasonics Sonochemistry 18: 888-94.

Gore, M. M., V. Kumar Saharan, D. V. Pinjari, P. V. Chavan, and A. B. Pandit. 2014. "Degradation of Reactive Orange 4 Dye Using Hydrodynamic Cavitation Based Hybrid Techniques." Ultrasonics Sonochemistry 21: 1075-82.

Guo, F., X. Huang, Z. Chen, H. Sun, and W. Shi. 2020. “Investigation of Visible-Light-Driven Photocatalytic Tetracycline Degradation via Carbon Dots Modified Porous $\mathrm{ZnSnO}_{3}$ Cubes: Mechanism and Degradation Pathway." Separation and Purification Technology 253: 117518

Joshi, R. K., and P. R. Gogate. 2012. "Degradation of Dichlorvos Using Hydrodynamic Cavitation Based Treatment Strategies." Ultrasonics Sonochemistry 19: 532-9.

Khan, M. H., H. Bae, and J. Y. Jung. 2010. “Tetracycline Degradation by Ozonation in the Aqueous Phase: Proposed Degradation Intermediates and Pathway." Journal of Hazardous Materials 181 (1-3): 659-65.

Kuldeep, R., and V. S. Moholkar. 2020. "Sulfadiazine Degradation Using Hybrid AOP of Heterogeneous Fenton/Persulfate System Coupled with Hydrodynamic Cavitation.” Chemical Engineering Journal 386: 121294.

Leng, L., L. Wei, Q. Xiong, S. Xu, W. Li, S. Lv, Q. Lu, L. Wan, Z. Wen, and W. Zhou. 2020. "Use of Microalgae Based Technology for the Removal of Antibiotics from Wastewater: A Review." Chemosphere 238: 1-14.

Li, H., S. Sun, H. Ji, W. Liu, and Z. Shen. 2020. "Enhanced Activation of Molecular Oxygen and Degradation of Tetracycline Over Cu-S4 Atomic Clusters." Applied Catalysis B: Environmental 272: 118966.

Manuel, C.-C., M. J. Fernández-Sanjurjo, F.-C. Gustavo, D. Fernández-Calviño, M. Arias-Estevez, A. Núñez-Delgado and E. Álvarez-Rodríguez. 2020. "Competitive Adsorption and 
Desorption of Three Tetracycline Antibiotics on Bio-Sorbent Materials in Binary Systems." Environmental Research 190: 110003.

Niu, L., G. Zhang, G. Xian, Z. Ren, T. Wei, Q. Li, Y. Zhang, and Z. Zou. 2021. "Tetracycline Degradation by Persulfate Activated with Magnetic $\gamma-\mathrm{Fe}_{2} \mathrm{O}_{3} / \mathrm{CeO}_{2}$ Catalyst: Performance, Activation Mechanism and Degradation Pathway." Separation and Purification Technology 259: 118156.

Sarvothaman, V. P., A. T. Simpson, and V. V. Ranade. 2019. "Modelling of Vortex Based Hydrodynamic Cavitation Reactors.” Chemical Engineering Journal 377: 119639.

Simpson, A., and V. V. Ranade. 2018. "Modelling of Hydrodynamic Cavitation with Orifice: Influence of Different Orifice Designs." Chemical Engineering Research and Design 136: 698-711.

Thanekar, P., P. R. Gogate, Z. Znak, Y. Sukhatskiy, and R. Mnykh. 2021. "Degradation of Benzene Present in Wastewater Using Hydrodynamic Cavitation in Combination with Air." Ultrasonics Sonochemistry 70: 105296.

Vivek, V. R., P. S. Varaha, S. Alister, S. Nagarajan, and I. Group. 2021. "Scale-Up of Vortex Based Hydrodynamic Cavitation Devices: A Case of Degradation of Di-chloro Aniline in Water." Ultrasonics Sonochemistry 70: 105295.

Wang, J., and R. Zhuan. 2020. "Degradation of Antibiotics by Advanced Oxidation Processes: An Overview." Science of the Total Environment 701: 1-15.

Wang, B., R. Zhang, and X. Lian. 2019. A Swirling Vortex Cavitation. China Patent. ZL201821724174.2.

Wang, C., R. Jin, Z. He, Y. Qiao, Y. Wang, K. Wang, Y. Lu, X. Wang, and D. Liu. 2020. “A New Water Treatment Technology for Degradation of $\mathrm{B}[\mathrm{a}] \mathrm{A}$ by Hydrodynamic Cavitation and Chlorine Dioxide Oxidation." Ultrasonics Sonochemistry 61: 104834.

Wang, H., J. Zhang, P. Wang, L. Yin, Y. Tian, and J. Li. 2020. "Bifunctional Copper Modified Graphitic Carbon Nitride
Catalysts for Efficient Tetracycline Removal: Synergy of Adsorption and Photocatalytic Degradation." Chinese Chemical Letters 31: 2789-94.

Wang, J., X. Wang, P. Guo, and J. Yu. 2011. "Degradation of Reactive Brilliant Red K-2BP in Aqueous Solution Using Swirling JetInduced Cavitation Combined with $\mathrm{H}_{2} \mathrm{O}_{2}$." Ultrasonics Sonochemistry 18: 494-500.

Wang, X., J. Jia, and Y. Wang. 2017. "Combination of Photocatalysis with Hydrodynamic Cavitation for Degradation of Tetracycline." Chemical Engineering Journal 315: 274-82.

Wang, X., J. Wang, P. Guo, W. Guo, and G. Li. 2008. “Chemical Effect of Swirling Jet-Induced Cavitation: Degradation of Rhodamine B in Aqueous Solution." Ultrasonics Sonochemistry 15: 357-63.

Wei, Z., J. Liu, and W. Shangguan. 2020. "A Review on Photocatalysis in Antibiotic Wastewater: Pollutant Degradation and Hydrogen Production." Chinese Journal of Catalysis 41: 1440-50.

Yang, S., Y. Feng, D. Gao, X. Wang, N. Suo, Y. Yu, and S. Zhang. 2021. "Electrocatalysis Degradation of Tetracycline in a ThreeDimensional Aeration Electrocatalysis Reactor (3D-AER) with a Flotation-Tailings Particle Electrode (FPE): Physicochemical Properties, Influencing Factors and the Degradation Mechanism." Journal of Hazardous Materials 407: 124361.

Zhang, X., H. Yang, and Z. Li. 2007. "Relationship Between Strength of Hydrodynamic Cavitation and Amount of Induced Hydroxyl Radical." Journal of Chemical Industry and Engineering (China) 58: 27-32.

Zhu, X., F. Guo, J. Pan, H. Sun, L. Gao, J. Deng, X. Zhu, and W. Shi. 2021. "Fabrication of Visible-Light-Response Face-Contact $\mathrm{ZnSnO}_{3} @ g$ $\mathrm{C}_{3} \mathrm{~N}_{4}$ Core-Shell Heterojunction for Highly Efficient Photocatalytic Degradation of Tetracycline Contaminant and Mechanism Insight." Journal of Materials Science 56: 4366-4379. 